\title{
BMJ Open Randomised controlled trial using a theory-based $m$-health intervention to improve physical activity and sleep health in adults: the Synergy Study protocol
}

\author{
Beatrice Murawski, ${ }^{1,2}$ Ronald C Plotnikoff,, ${ }^{1,3}$ Anna T Rayward, ${ }^{1,2}$ \\ Corneel Vandelanotte, ${ }^{4}$ Wendy J Brown, ${ }^{5}$ Mitch J Duncan ${ }^{1,2}$
}

To cite: Murawski B,

Plotnikoff RC, Rayward AT, et al. Randomised controlled trial using a theory-based m-health intervention to improve physical activity and sleep health in adults: the Synergy Study protocol. BMJ Open 2018;8:e018997. doi:10.1136/ bmjopen-2017-018997

\section{- Prepublication history for} this paper is available online. To view these files, please visit the journal online (http://dx.doi. org/10.1136/bmjopen-2017018997).

Received 8 August 2017 Revised 18 0ctober 2017 Accepted 14 November 2017

Check for updates

For numbered affiliations see end of article.

Correspondence to Dr Mitch J Duncan; mitch.duncan@newcastle. edu.au

\section{ABSTRACT}

Introduction There is a need to reduce physical inactivity and poor sleep health in the adult population to decrease chronic disease rates and the associated burden. Given the high prevalence of these risk behaviours, effective interventions with potential for wide reach are warranted.

Methods and analysis The aim of this two-arm RCT will be to test the effect of a three month personalised mobile app intervention on two main outcomes: minutes of moderate-to-vigorous-intensity physical activity and overall sleep quality. In addition, betweengroup changes in health-related quality of life and mental health status will be assessed as secondary outcomes. The pre-specified mediators and moderators include social cognitive factors, the neighbourhood environment, health (BMI, depression, anxiety, stress), sociodemographic factors (age, gender, education) and app usage. Assessments will be conducted after three months (primary endpoint) and six months (follow-up). The intervention will provide access to a specifically developed mobile app, through which participants can set goals for active minutes, daily step counts, resistance training, sleep times and sleep hygiene practice. The app also allows participants to log their behaviours daily and view progress bars as well as instant feedback in relation to goals. The personalised support system will consist of weekly summary reports, educational and instructional materials, prompts on disengagement and weekly facts.

Ethics and dissemination The Human Research Ethics Committee of The University of Newcastle, Australia granted full approval: H-2016-0181. This study will assess the efficacy of a combined behaviour intervention, mechanisms of behaviour change and gather high-quality process data, all of which will help refine future trials. Dissemination of findings will include publication in a peer-reviewed journal and presentation at national or international conferences. Participants will receive a plain English summary report of results.

Trial registration number ACTRN12617000376347; Preresults.

\section{Strengths and limitations of this study}

- No previous studies have tested the efficacy of a mobile intervention targeting physical activity and sleep in combination in a randomised waitlist controlled trial in physically inactive adults reporting poor quality sleep, who do not have a sleep disorder.

- Outcome data will contribute to the knowledge relating to chronic disease prevention through multi health behaviours using a mobile intervention with wide reach.

- Findings will facilitate the future design of technology-based multi health behaviour change interventions.

- Limitations include the lack of an intervention arm, which receives only the physical activity or only the sleep intervention.

- Remotely delivered (mobile) interventions are known to have high attrition rates.

\section{BACKGROUND}

Engaging in sufficient physical activity and maintaining good sleep health are two lifestyle behaviours that significantly reduce the risk of all-cause mortality, ${ }^{12}$ cardiovascular disease, ${ }^{34}$ and type 2 diabetes. ${ }^{56}$ Sufficient physical activity is the accumulation of at least 150 minutes of moderate-intensity or 75 minutes of vigorous-intensity physical activity per week. ${ }^{7}$ Good sleep health is characterised by duration, quality and timing of sleep that leave a person satisfied with their sleep and alert during the day. ${ }^{8}$ Internationally, up to $32 \%$ of adults are insufficiently physically active, ${ }^{9}$ and up to $29 \%$ report sleeping $<6$ hours, ${ }^{10} 24 \%$ report poor quality sleep, ${ }^{11}$ and $>50 \%$ report inconsistent bed and wake times, the latter of which are indicators of poor sleep health. ${ }^{12}$ There is no global estimate of the percentage of adults who report both insufficient physical activity 
and poor sleep health. However, evidence suggests that individuals with poor sleep health also report lower levels of physical activity. ${ }^{13} 14$ Thus, interventions which target both behaviours have the potential to make meaningful contributions to public health.

Multiple lifestyle behaviour interventions produce greater reductions in the risk of poor health than interventions that target a single behaviour. ${ }^{15}$ Moreover, physical activity and sleep have a bidirectional relationship, ${ }^{16}$ in which physical activity improves indicators of sleep health (eg, sleep quality) and good sleep health is associated with greater levels of physical activity. ${ }^{17}$ Interventions targeting both behaviours simultaneously may capitalise on this reciprocal relationship to produce larger increases in both behaviours. ${ }^{18}$ Previous reviews of multiple behaviour interventions however, have not identified any studies that specifically target changes in both physical activity and sleep health and tested the efficacy of this approach in a randomised controlled trial. ${ }^{19-21}$

Non-pharmacological sleep interventions (eg, Cognitive Behavioural Therapy for Insomnia) frequently promote sleep hygiene, ${ }^{22}$ using a set of self-regulatory strategies that help to promote good sleep health, but details of behaviour change techniques (BCT) to support changes in sleep hygiene behaviours, such as regular physical activity or stress management, are usually not reported. ${ }^{2324}$ Without providing the necessary guidance to promote behaviour change, it is unlikely that such education-only interventions produce changes in behaviour, as education-only interventions are known to be less effective than those that are combined with additional self-regulation strategies.$^{25}$ Furthermore, multiple health behaviour change interventions need to implement BCT that are specific to each behaviour to produce greater changes in targeted behaviours. ${ }^{26}$ Interventions targeting physical activity and sleep in combination therefore need to provide behaviour-specific intervention strategies to maximise change and harness the potentially synergistic effects between physical activity and sleep.

Reviews of the evidence suggest theory-based interventions are more effective in changing behaviour than interventions that do not use a theoretical approach. ${ }^{27}$ Theoretical models provide important guidance for the development of behaviour change interventions, aiming for the uniform operationalisation of cognitive and behavioural determinants. Social Cognitive Theory (SCT) is one of the most widely used theories in health behaviour research. ${ }^{28}$ SCT aids the conceptual understanding of behaviour change, as it accounts for the interactions between individual and environmental processes that either facilitate or impede behaviour change. ${ }^{29}$ This is particularly relevant when targeting both physical activity and sleep health, since individual as well as environmental factors are known to influence both behaviours. ${ }^{30} 31$ SCT has guided the development of numerous physical activity interventions and its constructs are strongly associated with physical activity, ${ }^{31}{ }^{32}$ but there is only limited understanding of social cognitive factors in relation to sleep health. ${ }^{30}$ However, it may be useful to apply social cognitive frameworks to better understand mechanisms of adult sleep health, since sleep is affected by factors at both the individual (eg, self-efficacy to change sleep hygiene behaviours) and environmental level (eg, sleep environment, neighbourhood factors).

Due to the high prevalence of people who report either being insufficiently active or meeting indicators of poor sleep health, there is a need for broad reaching interventions. Because smartphone ownership is growing steadily, with approximately $80 \%$ of the population owning a device ${ }^{33}$ intervention delivery entailing this medium is likely to be accessible, affordable and conveniently integrated into daily life.

This study aims to test: (1) the efficacy of an app-based intervention to improve physical activity and sleep quality (as primary outcomes) and health-related quality of life and mental health status (as secondary study outcomes), relative to a waitlist control; (2) the mediating role of social cognitive factors and app usage in behaviour change; and (3) health (BMI, depression, anxiety, stress), sociodemographic factors (age, gender, education) and the neighbourhood environment as potential moderators of intervention efficacy.

\section{METHODS}

This trial was registered prospectively (pre-results) on the Australian New Zealand Clinical Trials Registry (ANZCTR Registration Number: ACTRN12617000376347; Universal Trial Number: U1111-1186-6588). The conduct and reporting of the trial will follow CONSORT guidelines, ${ }^{34}$ and the CONSORT-EHEALTH check-list. ${ }^{35}$ Full ethical approval was obtained from the Human Research Ethics Committee (HREC) of The University of Newcastle, Australia (Approval Number: H-2016-0181).

\section{Study design}

A two-arm randomised controlled (superiority) trial with a combined physical activity and sleep intervention and a waitlist control group, with assessments conducted at zero months (baseline), three months (primary endpoint) and six months (follow-up).

\section{Recruitment}

Digital and print-based advertising will be used to recruit nationwide in Australia. Recruitment for both intervention arms commenced in May 2017 and will conclude once sample size requirements are achieved $(n=160$, refer to power and sample size section). Social media advertising will be used to recruit in social media networks (eg, Twitter, Facebook) using target audiences that match inclusion criteria (ie, age, living in Australia). Electronic and print-based advertising will include magazines and newspapers with state-wide reach. All recruitment materials will provide contact details and a link to the consent form and eligibility survey. Due to the remote delivery of the intervention in combination with self-report based 
assessments, participants will not be required to visit the research centre.

\section{Exclusion criteria}

Individuals who meet any of the following criteria will not be eligible to participate:

- not residing in Australia;

- not being between 18 and 55 years old;

- reporting a height and weight that is not consistent with a BMI between 18.5 and 35;

- accumulating more than 90 minutes of moderate/ vigorous physical activity per week;

- rating their sleep-quality (over the past month) as fairly good or very good;

- currently pregnant or having given birth in the past 12 months;

- having a condition that would make it unsafe or limits their ability to increase activity levels or change sleep behaviours;

- having a diagnosed sleep disorder (chronic insomnia, sleep apnoea, sleepwalking, narcolepsy, restless legs syndrome, etc);

- currently consuming hypnotics (sleep inducing medication);

- being employed in any night shift work;

- planning frequent travel (once a month or more often) to a destination with a shift in time zone by more than three hours during the intervention period;

- currently using a self-monitoring system or device to track or log physical activity or sleep (this includes non-device assisted applications); and

- not having access to an internet-enabled iOS (Apple) or Android smartphone or tablet.

Interested participants who indicated already using a self-monitoring system or tracking device were excluded to avoid the potentially confounding effect that the use of a self-monitoring system or device may have on behaviour, as most popular health apps or the trackers themselves frequently implement a variety of behaviour change strategies. $^{36} 37$

\section{Study procedure}

Eligible participants will be contacted via Email and welcomed into the study. Participants will be asked to complete online surveys assessing primary and secondary outcomes, potential mediators/moderators and socio-demographics at three time points. Figure 1 illustrates the flow of participants throughout the trial.

All online surveys will be administered using Qualtrics (Provo, Utah). If specified screening criteria are not met, participants will be advised via text displayed at the end of their survey and further contact will only be made where ambiguous responses require clarification. Ineligible participants will also receive a link providing free and unlimited access to the public version of the Balanced app. ${ }^{38}$

Participants will receive an Email with a unique password-protected link to their survey at each assessment point. Each person who has completed their baseline survey will be randomly allocated to one of two groups. Participants allocated to the intervention group will be mailed a pedometer, tool sheets, login details and instructions for download and installation of the 'Balanced' smartphone app in the form of a participant handbook. The initial Balanced app was specifically developed for scientific purposes and is described in more detail elsewhere.$^{38}$ It originally consisted of three separate categories, one for physical activity (active minutes), one for inactivity (hours and minutes of sitting) and one for sleep (bed and wake times and sleep quality rating). As part of the modifications to the previous app, the physical activity component of the app was revised to include daily steps and resistance training in addition to minutes of moderate-to-vigorous intensity physical activity; and the sleep component was revised to include sleep hygiene in addition to sleep times and sleep quality. The sitting behaviour category was removed for use in the Synergy study, as no specific strategies to reduce sitting time will be provided in this study and because the objective will be to promote improvements in moderate-to-vigorous intensity physical activity and sleep health. App content was modified based on participant feedback (process evaluation and semi-structured interviews) as part of the Balanced study, ${ }^{38}$ while design and aesthetics from the original version were retained. The main advance of the modified version lies in its increased level of tailoring using personal as opposed to the previously standardised goals, which makes feedback on progress towards goals and goal achievement more personalised and meaningful for those in need to get engaged in healthy behaviour. ${ }^{39}$

Regular app use will be supported by an Email and text message-based support system (see table 1), which is initiated as soon as a participant has gained access to the app. All messaging will follow a standardised protocol that was designed under consideration of the specificity, timeliness and relevance of contents (see table 1), as those are valued components in mobile apps designed to change health behaviours. ${ }^{40}$ Following completion of their three month assessment, participants may continue to use the app as much or as little as they like, but the message-based support will no longer be provided.

\section{Intervention}

The intervention is composed of app and non-app components, with non-app components referring to any content of the intervention that is delivered via participant handbook, text message or Email. App components consist of educational resources, self-monitoring, goal-setting and feedback. Participants will have continuous access to the app throughout the intervention period. For the first three months, which is the time between baseline and the primary endpoint, these components will be complemented by a messaging system providing personalised feedback on progress towards goals, prompting goal review and prompting practice of the target behaviours. The messaging component will cease at the three months 


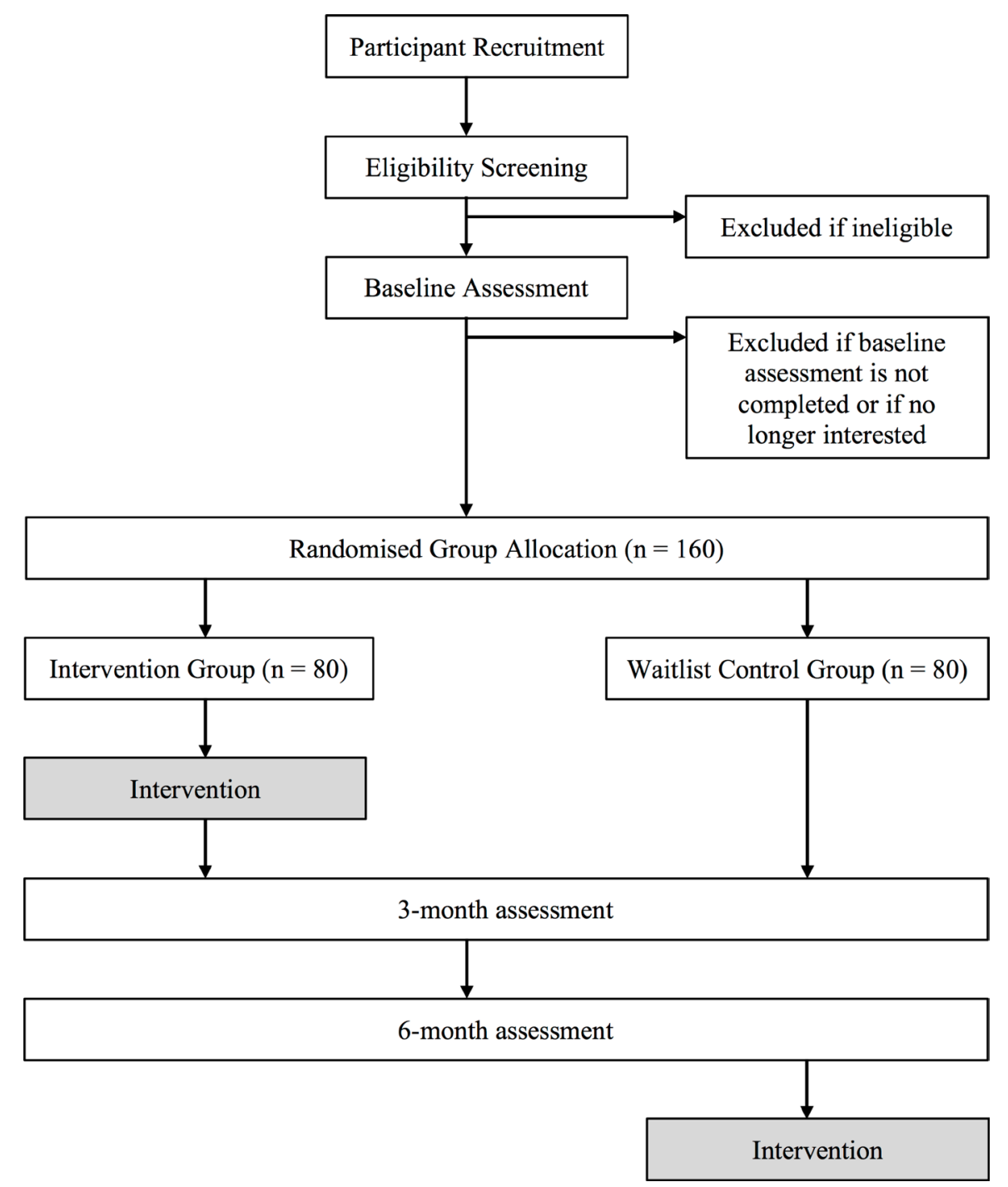

Figure 1 Flow of participants in the Synergy Study.

Table 1 Overview and content of message-based support service

Frequency

\begin{tabular}{|c|c|c|c|c|}
\hline \multirow[b]{2}{*}{ Delivery } & \multirow[b]{2}{*}{ Content } & \\
\hline & & weekly & monthly & as required \\
\hline \multirow[t]{4}{*}{ Email } & $\begin{array}{l}\text { General communication, survey reminders, notifications (eligibility, group } \\
\text { allocation) }\end{array}$ & & & $\mathrm{x}$ \\
\hline & Personalised weekly summary & $\mathrm{x}$ & & \\
\hline & Tool sheets (sent separately at weeks three, six and nine) & & $x$ & \\
\hline & $\begin{array}{l}\text { App usage reminder (Condition: if three consecutive Short Message Service } \\
\text { SMS prompts were unsuccessful in motivating participants to re-engage), only } \\
\text { if applicable }\end{array}$ & $\mathrm{x}$ & & \\
\hline \multirow[t]{2}{*}{ SMS } & Fact of the week & $\mathrm{x}$ & & \\
\hline & $\begin{array}{l}\text { Usage prompt (Condition: if non-usage occurred on at least four out of } \\
\text { seven days per week) }\end{array}$ & $\mathrm{x}$ & & \\
\hline $\begin{array}{l}\text { App-based } \\
\text { Prompts }\end{array}$ & $\begin{array}{l}\text { If enabled, a daily on-screen notification prompts participants to log data, if app } \\
\text { has not been used to self-monitor behaviour in }>24 \text { hours }\end{array}$ & & & $\mathrm{x}$ \\
\hline
\end{tabular}

The message-based support system will be delivered for the first 12 weeks of the intervention only. 
assessment, but participants will have continued access to the app. Following completion of the study, participants will be able to continue to access and use the app for an indefinite period, however will not be required to complete any further assessments as part of this study. The app will be available on both Android and Apple based operating systems. Table 2 provides an overview of intervention strategies used to operationalise the social cognitive constructs in the intervention. In brief, the key constructs included relate to a person's confidence (self-efficacy) in their capacity to define and follow a specific plan, the purpose of which is to experience a desired result in the face of situational or environmental (socio-structural) factors that either impede or facilitate progress, while the motivation to pursue results is regulated by perceptions regarding the personal benefit of the result in question and its perceived importance (outcome expectations and expectancies). ${ }^{29}$

\section{Educational resources}

App resources will consist of educational information about the importance of the two behaviours, basic instructions on how to change each behaviour and guidance for app use (eg, how to interpret traffic lights and progress graphs). This content will provide participants with knowledge on the health benefits of each behaviour, the current national guidelines for physical activity and sleep and the importance of resistance training and incidental physical activity in addition to aerobic exercise, as well as the importance of all dimensions of sleep health (ie, sleep duration, sleep quality, sleep timing). Resources for sleep will consist of a comprehensive range of stimulus control and sleep hygiene recommendations based on summaries of the evidence. ${ }^{22}$ In addition to app content, participants will receive a total of three tool sheets (enclosed in the handbook), one tool sheet including goal-setting strategies ${ }^{41}$ for each behaviour, one that emphasises action planning (again, one for each behaviour) and one tool sheet with information and instructions adapted from publicly available resources for the practice of stress management techniques (ie, progressive muscle relaxation, deep breathing and mindfulness). ${ }^{42-44}$ All tool sheets will be distributed at outset along with the participant handbook, which includes a brief study summary, a personalised timeline including assessment dates as well as a comprehensive troubleshooting guide covering the most common problems that may occur when installing and using the app. Participants in the intervention group will receive their materials following completion of their baseline assessment and waitlist controls will receive an identical package following their 6 month assessment. In addition, during each month of the intervention, one tool will be promoted via Email to encourage utilisation of these resources. Goal-setting tool sheets will be sent in week three, followed by the action planning tool sheet in week six and the stress management tool sheet in week nine for each participant. The examples given within the tool sheets are framed in a way that encourages participants to tailor any strategies to their own situation and priorities (for example: Once I get fitter, I will finally be able to...). Individuals are instructed to set goals that are personally relevant and meaningful to promote initial engagement, but the goal-setting information provided will give reference to the recommended minimum of 150 minutes of moderate-intensity physical activity per week, ${ }^{7}$ and a sleep duration of seven to ninehours per night ${ }^{45}$ as overarching goals one should gradually work towards. Weekly summary reports however, will focus on individual progress in relation to the individual goals set by the participant. Each report will detail progress in the form of totals and averages for both behaviours (ie, active minutes, step count, resistance training sessions, bed and wake times, sleep hygiene, sleep quality), which will help participants understand how changes in the two behaviours are interrelated. Furthermore, participants will receive a weekly text message containing one of 12 educational and motivational facts relating to physical activity and sleep for better health (ie, the consequences of poor sleep health). Each fact message will also refer to the resources section available in the app and encourage people to use it.

\section{Self-monitoring}

Participants will be asked to recall minutes of moderateto vigorous-intensity physical activity, and participation in resistance training, and manually enter this into the app every day. Daily steps will be objectively measured using the pedometer (Yamax SW200, Eagle Farm, QLD) provided and manually entered by participants into the app. Participants will not be asked to return their pedometer.

Self-monitoring of sleep in the app will also be manually entered by participants. The sleep log consists of: bedtime (time of going to sleep), wake time (time of waking) and sleep quality (rating scale from zero to five where five indicates high sleep quality). As an additional feature, this section of the app allows participants to log which sleep hygiene behaviours they practised the previous day (figure 2). These include consumption of caffeine, alcohol, nicotine, excessive intake of fluids or heavy meals before bedtime, regulation of the impact of light, noise and temperature in the bedroom, use of light-emitting devices, regular exercise, maintenance of consistent sleep and wake times, having and following a bedtime routine, creating comfort (eg, proper pyjamas and bedding) and managing stress. ${ }^{22}$ Participants can self-monitor these behaviours at any time of the day and update this information as many times per day as they prefer. The current study uses a manual data entry method based on self-monitoring. This method was selected for use in the current trial due to financial restrictions and because the Balanced study did not observe any between-group differences (ie, manual entry vs. device-entered method (via Fitbit)) in physical activity or sleep outcomes, or in time to non-usage attrition. ${ }^{38}$ 


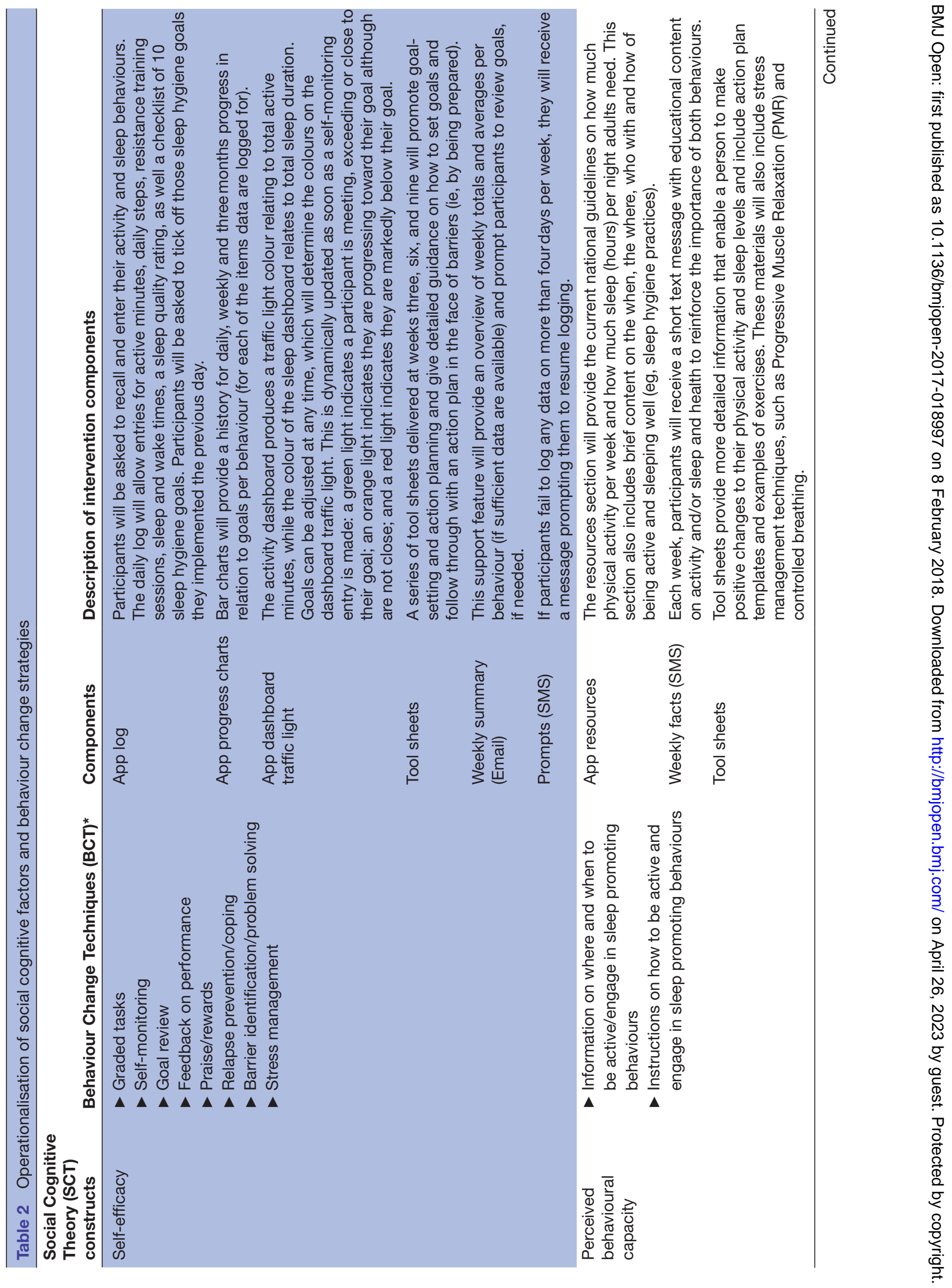




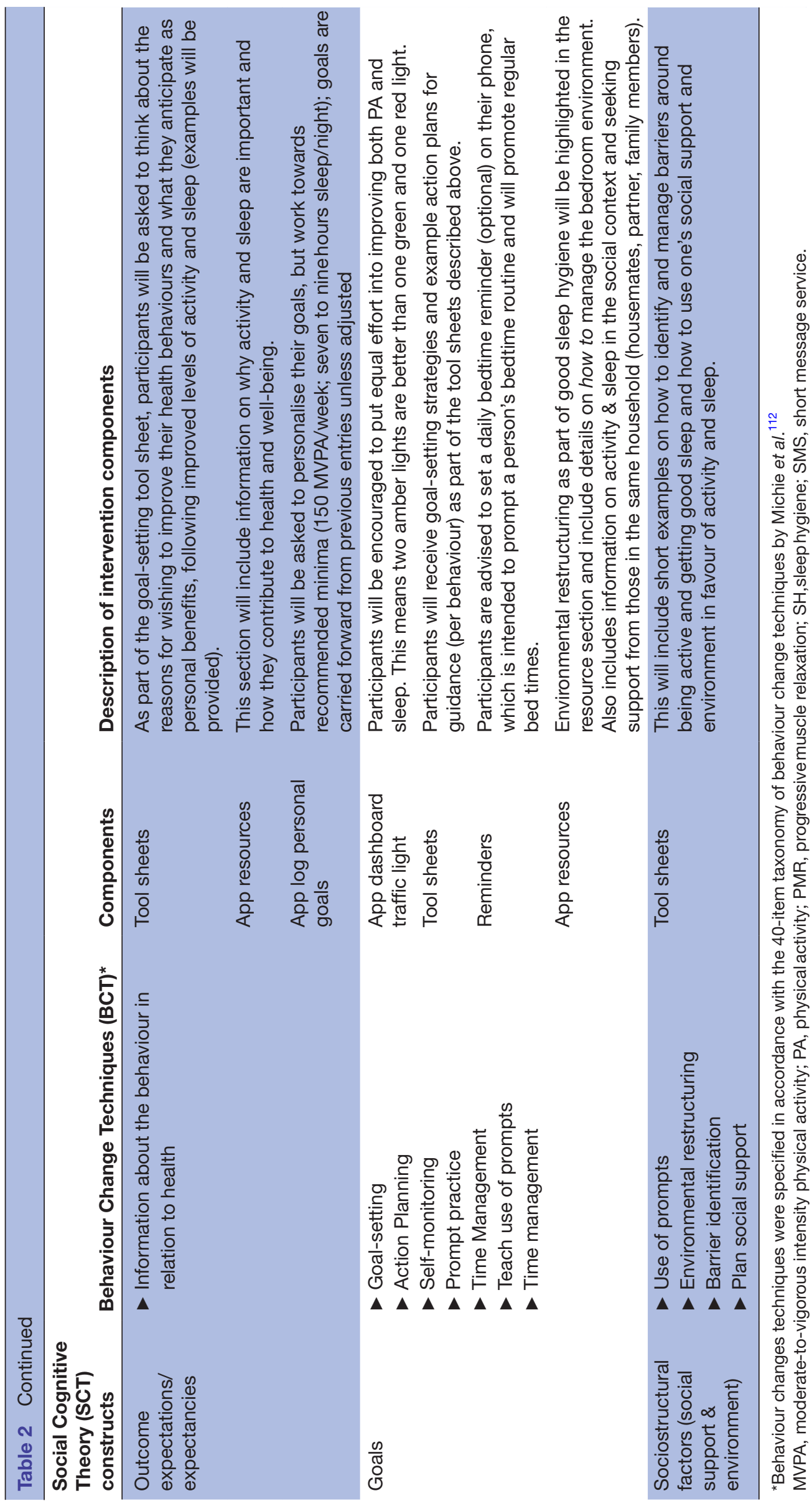




\section{Cancel}

Your Stats

Done

Caffeine

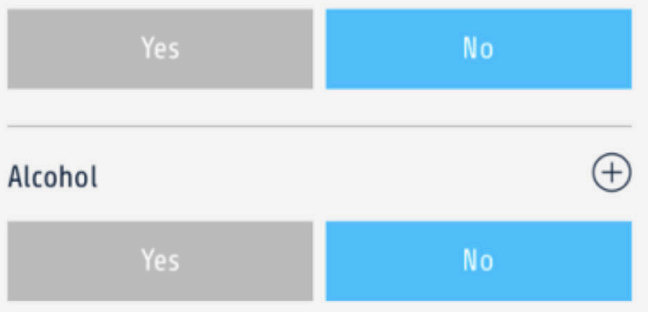

Food \& Drinks

Yes

No

Noise, Light \& Temperature

Yes

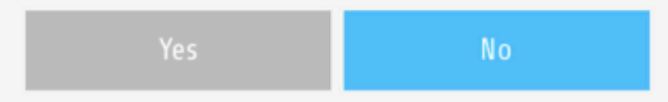

Light-Emitting Devices

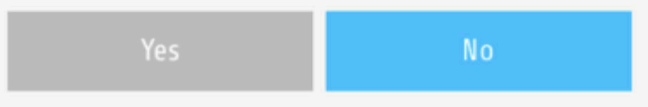

Physical Activity

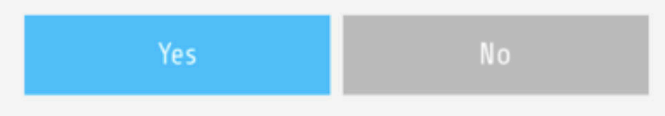

Sleep \& Waking Timing

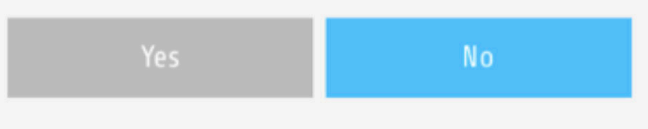

Bedtime Routine

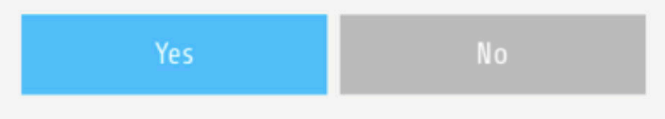

Bedroom Comfort

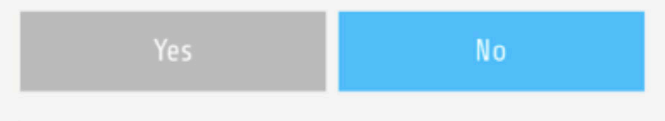

Stress Management
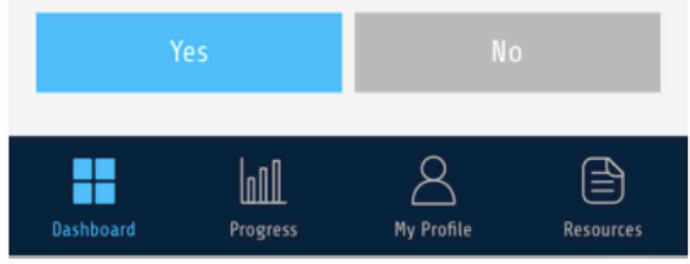

Figure 2 Sleep hygiene log items.

\section{Self-regulation}

App feedback on behaviour will be provided using graphical displays of logged behaviour in relation to the goals set by the participant (figure 3). Two types of graphical feedback are provided. There will be separate graphs for moderate-to-vigorous intensity physical activity, steps, resistance training, sleep duration, sleep quality, sleep timing and sleep hygiene. This information will provide a breakdown in the form of daily, one week and three month bar charts. The second graphical feedback to participants is via the dashboard which changes to one of three colours - green, orange and red in a traffic light system - to provide immediate feedback on participants' behaviour in relation to their goals on a daily basis (figure 3). The comparison of actual behaviour to goals based on a percentage of the goal achieved allows the use of consistent criteria across behaviours. This differs to the traffic light system originally used in Balanced, since process data from that study alluded to participants preferring to see this feedback based on goals rather than guidelines for each behaviour. ${ }^{38}$

As part of the goal review strategies, participants will be encouraged to evaluate their achievements in relation to goals and adjust their goals whenever needed. This will be facilitated by a personalised weekly summary of the previous week, delivered via Email, so that any reviews and adjustments of goals align with the most recent progress and foster self-efficacy. If a participant has logged data on less than four days per week (per behaviour), a text message will be sent to prompt practice. Likewise, once per week, if a participant only logs data for one behaviour, but not the other, a prompt will encourage him or her to engage in both behaviours equally.

\section{Waitlist control group}

Following enrolment and allocation, the waitlist control group will not receive any intervention materials and only be required to complete their three month and six month assessments. If the sixmonth assessment is completed, participants in this group will then receive full access to the intervention.

\section{Randomisation}

Participants will be randomly allocated to two groups (intervention or control) after having completed their baseline assessment. Opaque sealed envelopes $(n=80$ per group) will be prepared by BM using permuted block randomisation with block sizes of four and eight, following the procedures suggested by Doig et al. ${ }^{46}$ Once a participant has completed their baseline assessment, a researcher not associated with the study who is responsible for group allocation will open the envelope that is next in sequence and inform the project leader about the allocation outcome. Participants will be informed by the project leader and be sent a package containing study materials (ie, handbook and pedometer), if they have been allocated to the intervention group (participants in the waitlist control group will receive their study materials 


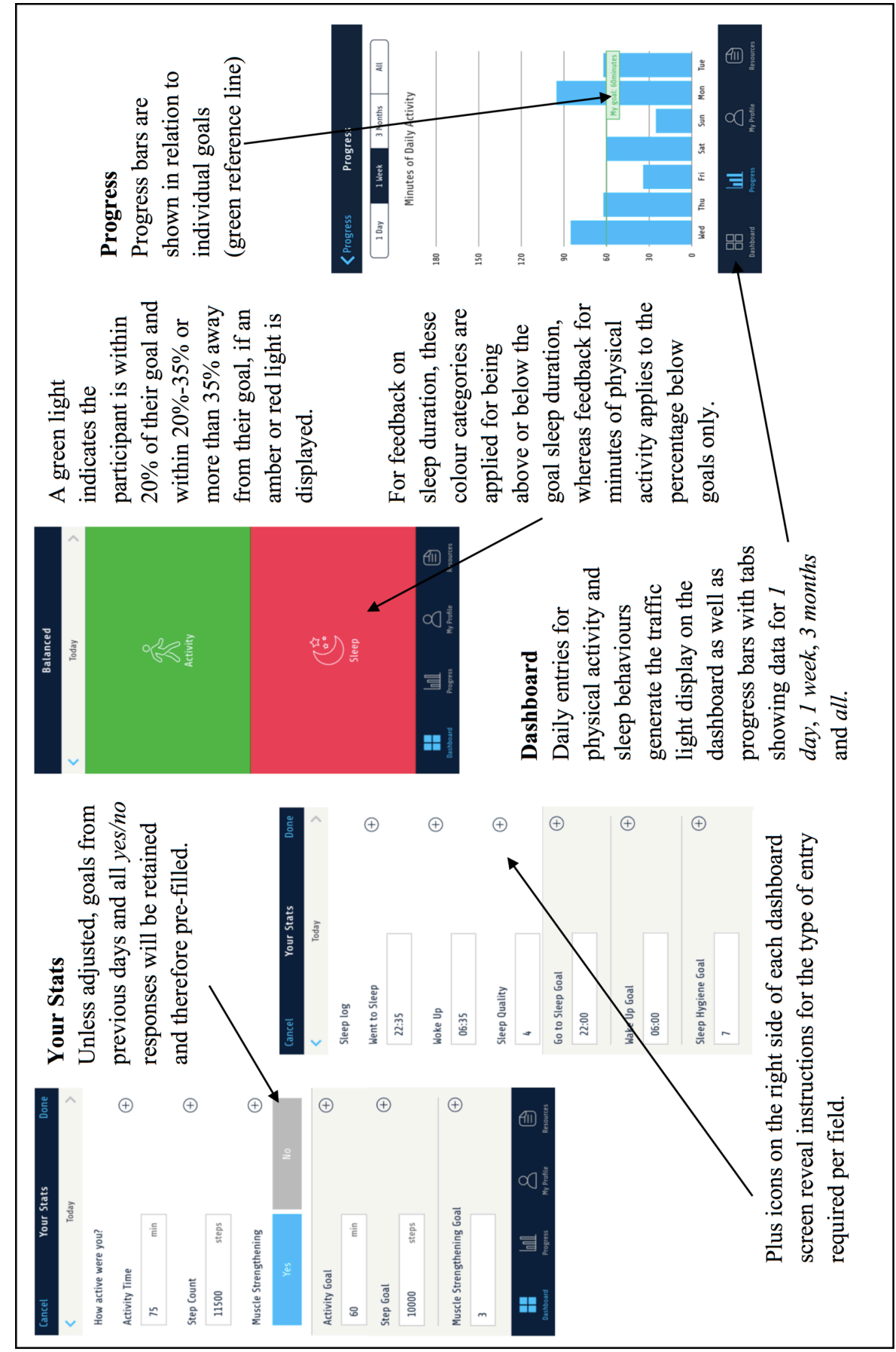

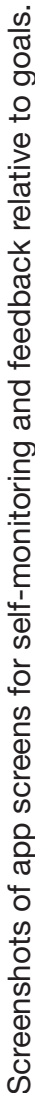

๓๐ 
after completing their six month assessment). The only exception for contravention with the allocation sequence will be made if family members or couples living in the same household enrol in the study, which would pose a high risk of contamination, especially between groups. For this reason, all individuals who are identified as members of the same household will be allocated to the same group. Neither the trial participants, nor the project lead (BM) will be blinded to group assignment.

\section{Outcome measures}

All measures will be assessed via online survey at baseline, three months, and six months, except for socio-demographics which will only be collected at baseline. The three month survey will further include process evaluation items that measure system usability and participant satisfaction (intervention group only). The two primary outcomes will be total minutes of moderate-to-vigorous physical activity and sleep quality. To increase adherence to scheduled assessments, participants who complete their survey will be entered into a draw for one of five $\$ 50$ shopping vouchers. This information will not be provided prior to enrolment and is not intended to function as an incentive for individuals to sign up to participate, but merely to promote adherence to assessment requirements. Table 3 provides a summary of outcome measures and assessment time points. All online surveys will be pilot-tested and locked prior to study commencement to prevent any changes from being made once the study is underway. All survey forms will be hosted on Qualtrics.

\section{Primary outcomes}

Physical activity

The Active Australia Questionnaire (AAQ) has demonstrated acceptable reliability $(\mathrm{rho}=0.64),{ }^{47}{ }^{48}$ is sensitive to change in interventions, ${ }^{49}$ and provides a measure of both the frequency and duration of moderate- and vigorous-intensity physical activity during the last week. This includes the total time spent in recreational walking and transport, moderate-intensity physical activity (eg, swimming, golfing), aerobic activity (eg, cycling, jogging) and vigorous gardening or yard work. Total minutes of moderate- and vigorous-intensity physical activity will be created by summing minutes of walking, moderate- and vigorous-intensity (weighted by two) physical activity. Although objective assessment methods may be used to measure physical activity, it was not deemed feasible in the current study due to financial and pragmatic issues.

\section{Sleep quality}

The Pittsburgh Sleep Quality Index (PSQI) consists of 19 items and seven component scores with scores ranging from zero to $21 .^{50}$ Items assess problems with seven different components of sleep health in the last 30 days. Higher scores indicate poorer sleep quality and a score above five is commonly used to indicate poor sleep quality. The current study will use the PSQI as a continuous score. The PSQI is the most frequently used self-report instrument in sleep research. ${ }^{51-53}$ The PSQI has demonstrated good reliability $(\alpha=0.83)$, is sensitive to change and has strong psychometric properties. ${ }^{50} 54$ The seven PSQI component scores consist of subjective sleep quality, sleep onset latency, sleep duration, habitual sleep efficiency, sleep disturbances, use of sleep medication and daytime dysfunction, all of which will be reported in addition to the total score. Although objective assessment methods (eg, polysomnography, accelerometry) are known to provide accurate measures of sleep, ${ }^{55}$ a global measure of subjective sleep quality will be used in this study to observe the perceived restorative effect of sleep, which is difficult to measure using objective methods. ${ }^{56}$

\section{Secondary outcomes}

Health-related quality of life

Poor sleep quality and inadequate sleep duration are independently associated with low health-related quality of life. ${ }^{57}$ The RAND-12 is a valid and reliable instrument ${ }^{58}$ that is widely used to assess multiple concepts of health, such as physical functioning, role limitations due to physical and emotional problems, social functioning, emotional well-being, energy/fatigue, pain as well as general perceptions of health. In addition to the RAND-12 scale, three additional items that make up the energy/ fatigue subscale in the 36 -item version of the RAND will be asked (eg, 'How much of the time during the past four weeks did you feel tired?'), so that this domain can be evaluated separately. This will allow improvements in energy and fatigue during the course of the intervention to be assessed.

\section{Depression, anxiety, and stress}

The effect of changes in physical activity and sleep on participants' severity of depression, anxiety and stress symptoms will be assessed using the Depression-Anxiety-Stress Scale (DASS-21). The DASS-21 is reported to have satisfactory levels of internal consistency for its total scale $(\mathrm{r}=0.93)$ as well as for its individual scales for depression $(\mathrm{r}=0.88)$, anxiety $(\mathrm{r}=0.82)$ and stress $(\mathrm{r}=0.90) .{ }^{59} \mathrm{In}$ addition, DASS-21 scores will be examined as a potential moderator of intervention efficacy.

\section{Resistance training}

Since the AAQ does not capture resistance training and because the Synergy Study will promote regular resistance training, the number and duration of resistance training sessions per week will be assessed using two items adapted from previous studies that assessed resistance training. ${ }^{60}$ One item will ask participants: 'In the last week, on how many days have you participated in muscle strengthening activities (including weight/resistance training)?' and 'What do you estimate was the total time (in hours/ minutes) that you spent doing muscle strengthening activities (incl. weight/resistance training) in the last week?' The original items were adapted by changing the recall period from the previous month to the last week to align with the recall period used in the AAQ. 
Table 3 Overview of outcome measures and assessment time points

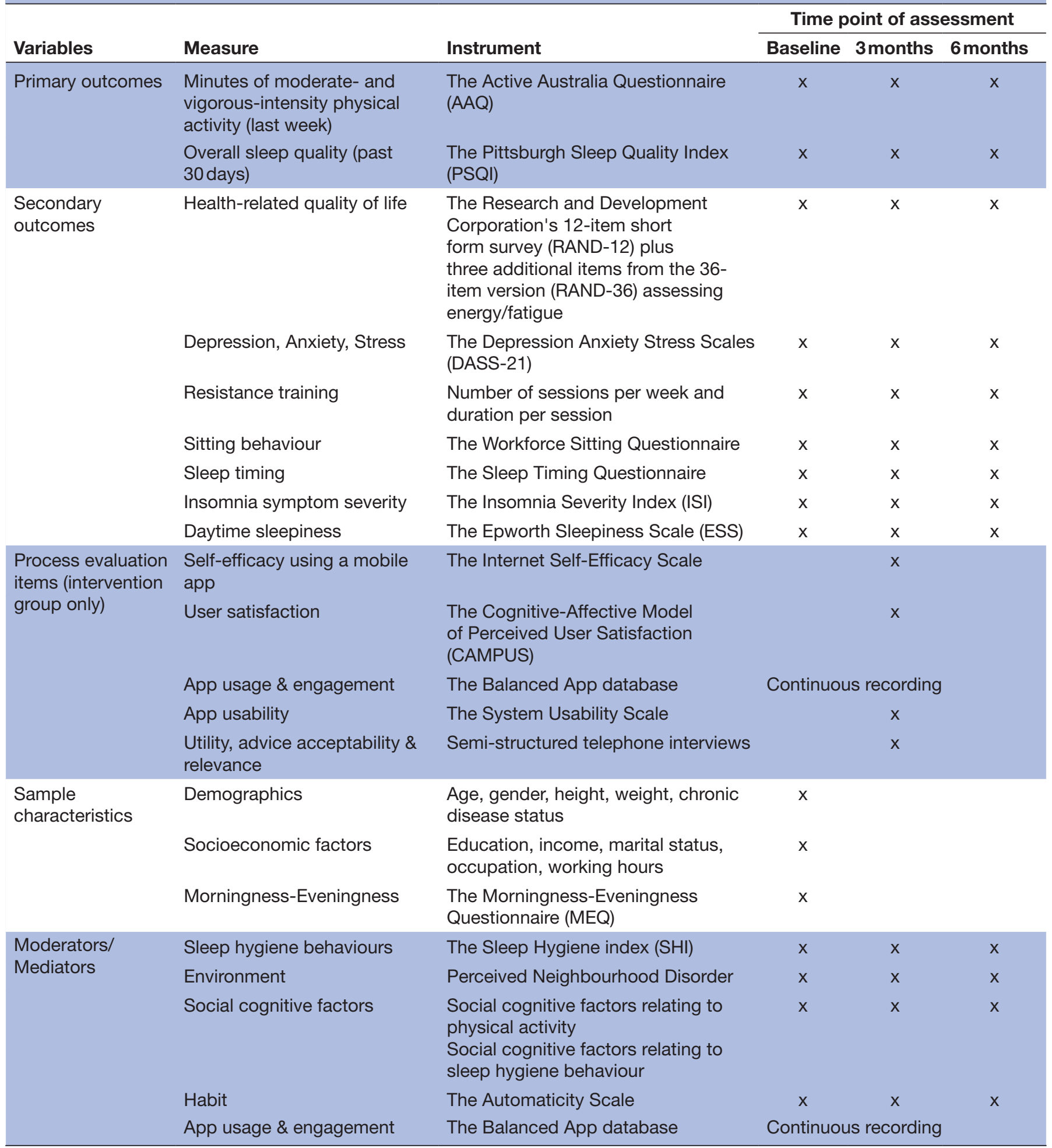

\section{Sitting time}

The Workforce Sitting Questionnaire (WFSQ) will provide a self-report measure of total domain-specific sitting time (over the last week), on workdays and non-workdays. ${ }^{61}$ Domains include sitting time accumulated at work, watching TV, using a computer, using transport and doing other leisure activities. The WFSQ captures sitting time across several domains with acceptable validity $(r=0.45)$ and reliability $(\mathrm{ICC}=0.63)$. Possible reductions in total sitting time may be a result of increased amounts of time allocated to light/incidental or moderate-to-vigorous-intensity physical activity. ${ }^{62}$

Sleep timing

A modified version of the validated Sleep Timing Questionnaire will be used to assess the variability in sleep and 
wake times on working days as well as non-working days. ${ }^{63}$ To minimise participant burden, the instrument used will only include items on the stability of usual bed and wake times, and the usual bed and wake times per se. Response options are categorical and scored on a scale from one to 11 with lower scores indicating less variability in bed or wake times (eg, $1=0-15 \mathrm{~min}$; $2=16-30 \mathrm{~min} ; 11=>4 \mathrm{hr}$ ).

\section{Insomnia severity}

The Insomnia Severity Index (ISI) is a valid and reliable instrument for measuring insomnia severity. ${ }^{64}$ It can be used to classify individuals as having no insomnia (0-7), sub-threshold insomnia (8-14), moderate clinical insomnia (15-21) or severe clinical insomnia (22-28). This index will measure the proportion of the sample with potentially severe, yet undiagnosed insomnia symptoms. While assessing the severity of sleep problems and the level of dissatisfaction with sleep a person can experience, the ISI also captures the extent to which the consequences of sleep problems manifest themselves in everyday life, for example "To what extent do you consider your sleep problem to interfere with your daily functioning (eg, daytime fatigue, mood, ability to function at work/daily chores, concentration, memory, mood, etc) currently?". Across a total of seven items, responses are scored on a five-point scale and summed to obtain a total score.

\section{Daytime sleepiness}

Daytime sleepiness is a further indicator of poor sleep health. It will be measured using the Epworth Sleepiness Scale (ESS), which assesses daytime sleepiness. This scale has demonstrated high internal consistency (Cronbach's alph $a=0.88$ ) and good reliability $(r=0.82)^{65}$ and consists of eight items that depict various situations in which a person could experience dozing off (eg, while sitting and reading or watching TV). Items are summed to calculate a total score from zero to 24 with higher scores indicating higher levels of daytime sleepiness.

\section{Process outcomes}

Internet self-efficacy

Participants' confidence in using the smartphone app will be assessed using an adaptation of the Internet Self-Efficacy Scale to capture participants' overall understanding of app software, confidence in gathering information using the app and learning to use the app, as well as the ability to troubleshoot and resolve app problems. ${ }^{66}$ Participants will rate their agreement using a total of eight statements (eg, 'I feel confident explaining why a task will not run on the smartphone/tablet') on a seven-point scale from strongly disagree to strongly agree.

\section{Perceived user satisfaction}

The Cognitive-Affective Model of Perceived User Satisfaction (CAMPUS) will be used to ask participants about thoughts and feelings associated with using the mobile app (Balanced). Using a seven-point scale ranging from strongly disagree to strongly agree, a total of 23 items enquire about participant opinion on the effects and aesthetics as part of the app design (15 items), its effectiveness and efficiency (five items) and the level of satisfaction experienced when using the app (three items) with the following semantic differentials: frustrated - contented, unhappy - gratified and sad - joyful. Items will be adapted to refer specifically to the Balanced app, for example 'I would consider my experience with using the Balanced app as innovative'. This instrument has demonstrated adequate levels of reliability and validity. ${ }^{67}$

\section{App usage}

Overall interaction with the app will be measured continuously throughout the study period by the app database, which records the time and date a self-monitoring entry was made and the actual value or response entered into the app. Analysis of usage patterns will include the number of self-monitoring entries made and the duration of self-monitoring throughout the intervention, similar to previous research. ${ }^{68}{ }^{69}$ These data will also be considered as a mediator of behaviour change in the intervention group.

\section{Usability of the app}

App usability will be assessed using the 10-item System Usability Scale, ${ }^{70}$ a valid and reliable tool that assesses participant satisfaction relating to the utility of websites on a five-point scale (items will be reworded for smartphone app usability). Higher total scores (range 1-100) relate to better usability. Example items include 'I would imagine that most people would learn to use this system very quickly.' or 'I needed the support of a technical person to be able to use this system'.

\section{Utility, advice acceptability and relevance of the app}

A participant sub-sample $(10 \%)$ will be determined by random selection for semi-structured telephone interviews, which will take place once all participants have completed their six month assessments. These interviews will contribute valuable information for process evaluation and include general personal feedback, desirable improvements and preferences relating to future use. As part of these interviews, participants will be asked about their perception of the app's usefulness to improve changes in self-efficacy levels (confidence) toward physical activity and sleep health, coping with potential impediments (barriers) to being more active and sleeping better, maintaining new routines/action plans and keeping it a priority to be more active and sleeping better. Finally, advice acceptability and relevance in terms of the content will be examined based on a previously used questionnaire. ${ }^{71}$

\section{Mediators and moderators}

\section{Social cognitive factors}

Testing social cognitive factors as potential mediators of intervention efficacy may provide insights into some of the underlying mechanisms of behaviour change, as observed in previous health behaviour interventions. ${ }^{72}$ 
Table 4 Social cognitive factors related to physical activity and sleep hygiene behaviours

\begin{tabular}{|c|c|c|}
\hline Construct & Items & Response anchors \\
\hline \multicolumn{3}{|l|}{ Physical Activity } \\
\hline Self-efficacy & 10 & $\begin{array}{l}\text { (1) not at all confident } \\
\text { (5) extremely confident }\end{array}$ \\
\hline $\begin{array}{l}\text { Perceived behavioural } \\
\text { capacity }\end{array}$ & 3 & $\begin{array}{l}\text { (1) never } \\
\text { (5) always }\end{array}$ \\
\hline Outcome expectations & 5 & $\begin{array}{l}\text { (1) strongly disagree } \\
\text { (7) strongly agree }\end{array}$ \\
\hline Outcome expectancies & 5 & $\begin{array}{l}\text { (1) not at all important } \\
\text { (4) extremely important }\end{array}$ \\
\hline Environment & 3 & $\begin{array}{l}\text { (1) strongly disagree } \\
\text { (5) strongly agree }\end{array}$ \\
\hline Social support & 2 & $\begin{array}{l}\text { (1) strongly disagree } \\
\text { (5) strongly agree }\end{array}$ \\
\hline Goals & 2 & $\begin{array}{l}\text { (1) no, not really } \\
\text { (7) strongly intend; and } \\
\text { (1) not at all motivated } \\
\text { (7) extremely motivated }\end{array}$ \\
\hline Action planning & 4 & $\begin{array}{l}\text { (1) no detailed plans } \\
\text { (7) detailed plans }\end{array}$ \\
\hline \multicolumn{3}{|c|}{ Sleep Hygiene Behaviours $(\mathrm{k}=9)$} \\
\hline Self-efficacy & 9 & $\begin{array}{l}\text { (1) not at all confident } \\
\text { (5) extremely confident }\end{array}$ \\
\hline $\begin{array}{l}\text { Perceived behavioural } \\
\text { capacity }\end{array}$ & 9 & $\begin{array}{l}\text { (1) never } \\
\text { (5) always }\end{array}$ \\
\hline Outcome expectations & 9 & $\begin{array}{l}\text { (1) strongly disagree } \\
\text { (7) strongly agree }\end{array}$ \\
\hline Outcome expectancies & 9 & $\begin{array}{l}\text { (1) not at all important } \\
\text { (4) extremely important }\end{array}$ \\
\hline Environment & 9 & $\begin{array}{l}\text { (1) strongly disagree } \\
\text { (5) strongly agree }\end{array}$ \\
\hline Social support & 9 & $\begin{array}{l}\text { (1) strongly disagree } \\
\text { (5) strongly agree }\end{array}$ \\
\hline Goals & 9 & $\begin{array}{l}\text { (1) no, not really } \\
\text { (7) strongly intend and }\end{array}$ \\
\hline Action planning & 9 & $\begin{array}{l}\text { (1) no detailed plans } \\
\text { (7) detailed plans }\end{array}$ \\
\hline
\end{tabular}

Each item per construct will refer to one of nine different sleep hygiene behaviours.

Constructs from Social Cognitive Theory ${ }^{29}$ will be assessed using partially modified items from previously developed scales, with distinct items per behaviour relating to the person's projections towards the occurrence of each behaviour over the next threemonths. The constructs of interest include self-efficacy, perceived behavioural capacity, outcome expectations and expectancies, goals, action planning and socio-structural factors including social support and the environment. Items are described in more detail below and table 4 summarises the number of items per behaviour per construct and lists response options for each item.

\section{Physical activity items}

For physical activity, a total of 34 items will be used to assess the social cognitive factors and a sum score will be calculated for each construct. Prior to asking these questions, participants will be advised that in the context of these questions "regular physical activity is defined as doing at least 150 minutes of moderate intensity physical activity each week. Moderate intensity can be described as any type of aerobic activity performed at a level where a person begins to lightly sweat, but can still carry on a conversation. This may feel different from one person to another."

\section{Self-efficacy}

Self-efficacy levels in the context of barriers will be assessed using a modified version of validated measures ${ }^{73}$ consisting of 10 items. Response choices for these items range from not at all confident (1) to extremely confident (5) and items share the same stem ('I am confident that I can participate in regular physical activity [...]'), followed by situations or circumstances that may impede regular engagement in physical activity (ie, "when I am a little tired, I am in a bad mood or feeling depressed, I have to do it by myself, it becomes boring, I can't notice any improvements in my fitness, I have many other demands on my time, I feel a little stiff and sore, the weather is bad, I have to get up early, even on weekends, I am on vacation').

\section{Behavioural capacity}

Participants will be asked how confident they are about having the capacity to engage in specific amounts and intensities of physical activity, using three statements ${ }^{74}$ and response options from never (1) to always (5). An example statement is: 'I can run or jog for 10 minutes without stopping.'

\section{Outcome expectations and expectancies}

Using five items per construct, a total of 10 items will assess participants' expectations and expectancies pertaining to perceived personal gains (outcome expectations) from engaging in regular physical activity, followed by the level of importance associated with these gains (outcome expectancies). On a five-point Likert scale (strongly disagree to strongly agree), participants will be asked first to indicate their level of agreement with one of five statements (adapted from Dewar, et $a l^{75}$ ) relating to perceived benefits of regular engagement in physical activity (eg, 'Being physically active can reduce my risk for some illnesses and diseases (eg, heart disease, diabetes, some cancers, etc)') and then rate the value this would have for themselves (eg, 'How important is reducing your risk for illness and disease?') on a four-point Likerttype scale (not at all important to 'extremely important'). One sum score will be calculated for outcome expectations and one for outcome expectancies.

\section{Social support}

The role a person's social network plays in influencing physical activity participation will be assessed by asking 
participants about their level of agreement (on a sevenpoint scale from strongly disagree to strongly agree) with two items that were previously modified for use in the context of a physical activity intervention ${ }^{76}$ : 'People in my social network are likely to help me participate in regular physical activity.' and 'I feel that someone in my social network will provide me with the support I need to get regular physical activity'.

\section{Environment}

The impact a person's built and natural environment has on physical activity engagement will be measured using three items from the IPAQ environmental module ${ }^{77}$ that are answered on a five-point Likert scale. This scale has shown acceptable levels of reliability with intra-class correlations ranging from 0.36 to 0.98 . The three items are 'There are sidewalks on most of the streets in my local area', 'There are many interesting things to look at while walking my local area.' and 'My local area has several free or low-cost recreation facilities, such as parks, walking trails, biking paths, playgrounds, and recreation centres'. Higher total scores correspond with an environment that facilitates physical activity, whereas lower scores indicate environmental impediments that may have a negative influence on physical activity levels.

\section{Goals}

To further assess the motivational mechanisms that drive progress towards goal attainment, ${ }^{29}$ participants will be asked to indicate the extent to which they intend to be active on a regular basis using two adapted items: 'Do you intend to do regular physical activity over the next three months?"75 and 'How motivated are you to do regular physical activity over the next three months? ${ }^{38}$ that are answered using seven-point Likert-type response choices ranging from no, not really (1) to strongly intend (7) and not at all motivated (1) to extremely motivated (7), respectively. For both items, higher scores indicate greater strength of goals and the two scores will be summed to obtain a total score for goals.

\section{Planning}

Plans concerning 'when', 'where', 'how' and 'what kind' of physical activity participants will engage in will be assessed using a previously modified scale ${ }^{79}$ that consists of four respectively worded items, where higher scores are interpreted as more detailed planning (no plans (1) - detailed plans (7)).

\section{Sleep hygiene items}

To assess the same constructs as above in the context of sleep hygiene practice, a total of 72 items were developed using partially modified scales that were previously used to assess social cognitive factors in the context of other health behaviours (ie, physical activity, diet). ${ }^{73} 75$ Each scale will query each of the following nine sleep hygiene practices: (1) avoiding caffeinated beverages (coffee, tea, energy drinks, etc) in the late afternoon or right before bedtime, (2) avoiding nicotine right before bedtime,
(3) avoiding alcohol right before bedtime, (4) exercising regularly, (5) reducing stress levels, (6) reducing the impact of noise and nuisance in the bedroom, (7) keeping sleep and wake times consistent, (8) avoiding daytime naps and (9) avoiding the use of technological devices (eg, phone, TV, laptop, etc) right before bedtime or in bed. To avoid overburdening participants, each construct will be assessed using a single item per sleep hygiene behaviour. Thus, each social cognitive scale will have nine items. Each scale will be scored as the sum of the nine sleep hygiene items, with a higher sum score indicating improvement. The environment construct however, will not be included for sleep hygiene behaviours, as this is already captured as part of the perceived neighbourhood disorder questionnaire described below.

\section{Self-efficacy}

Items assessing self-efficacy relating to sleep hygiene will be answered on a five-point Likert-type scale (not at all confident to extremely confident) using the commonly used stem 'I can $[\ldots]^{80}$ in connection with each of the nine sleep hygiene behaviours (eg, '[...] avoid alcohol right before bedtime', '[...] reduce the impact of noise and nuisance in my bedroom', etc).

\section{Behavioural capacity}

Participants will be asked to rate (never (1) to always (5)) their perceived behavioural capacity of making various choices in favour of keeping good sleep health using 'Whenever I have the opportunity to [...]' as a stem. For example, 'Whenever I have the opportunity to use technological devices right before bedtime or in bed, I know how to avoid or remove them.' These items were adapted from previously used scales ${ }^{75}$ with a focus on situations that challenge the reinforcement of making healthy dietary choices. In the context of avoiding behaviours that do not promote good sleep health, behavioural capacity can be thought of as a function of inhibitory control. ${ }^{81}$

\section{Outcome expectations and expectancies}

Similar to the scales described above for physical activity, those for sleep hygiene will be built on two single stems per sleep hygiene behaviour adapted from previous studies: 'For me, (keeping consistent sleep and wake times) would help me sleep better. ${ }^{73}$ and 'How important is it to (eg, keep sleep and wake times consistent) to sleep well?. ${ }^{75}$ The outcome expectations items are answered on a seven-point Likert scale and the outcome expectancies items are answered on a four-point scale ranging from not at all important (1) to extremely important. Sum scores will be reported separately for each of the two constructs.

\section{Social support}

To assess social support as a socio-structural factor that may or may not have a facilitating effect on sleep hygiene practice, the commonly used stem 'Most people who are important to me would encourage me to (eg, reduce my stress levels). ${ }^{78} 81$ will be used with response choices ranging from strongly disagree (1) to strongly agree (5). 


\section{Goals}

The extent to which people 'intend to [...]' practice sleep hygiene behaviours will be measured using a seven-point Likert-type scale (from no, not really to strongly intend) with higher scores indicating stronger goals. This item was used previously in a sleep hygiene context. ${ }^{81}$

\section{Planning}

To test participants' plans with regards to practising good sleep hygiene, each of the nine items assessing this construct will ask if a person has planned 'where, when and how' to avoid caffeine, avoid nicotine, avoid alcohol, exercise regularly, reduce their stress levels, minimise the impact of noise and nuisance in their bedroom, keep their sleep and wake times consistent, avoid daytime naps and avoid using technological devices right before bedtime or in bed. While previous studies ${ }^{79}$ have used four separate items to assess planning to engage in the behaviour ('when', 'where', 'how' and 'what kind' of behaviour x), these were collapsed into one item per sleep hygiene behaviour to reduce response burden.

\section{Automaticity}

Habits relating to lifestyle behaviours are non-conscious processes, which can act as determinants of behaviour and may even regulate behaviour independently of changes in conscious processes such as implementation intentions (goals). ${ }^{82}$ The role that behavioural automaticity plays in the context of physical activity and sleep behaviours, respectively, will be taken into account using one item from the Automaticity Index per sleep hygiene behaviour (nine items), ${ }^{83}$ and all four items of the index relating to physical activity (13 items in total), for example: 'Reducing the impact of noise in my bedroom is something I do automatically', 'Exercise is something I do without thinking'.

\section{Sleep hygiene}

Sleep hygiene will be assessed to measure changes in sleep hygiene behaviour using the 13-item Sleep Hygiene Index (SHI) developed by Mastin et al. ${ }^{84}$ Higher global scores indicate poorer sleep hygiene behaviour, but there is no cut-off to label categories of sleep hygiene engagement. This instrument demonstrates acceptable internal consistency $(\alpha=0.66)$ and test-retest reliability $(r=0.71$, $\mathrm{P}<0.01) .{ }^{84}$ Importantly, the SHI shows positive correlations $(r=0.48)$ with both the global scores $(\mathrm{P}<0.01)$ and the component scores $(\mathrm{P}<0.05$ or less $)$ of the Pittsburgh Sleep Quality Index. ${ }^{84}$ Items are answered using a fivepoint Likert-type scale from never (1) to always (5).

\section{Environment}

Perceptions about the order or disorder within a person's physical and/or social environment (ie, neighbourhood peacefulness, safety, cleanliness) can have a significant influence on physical activity levels and the quality and duration of sleep. ${ }^{85-87} \mathrm{~A}$ person's neighbourhood environment can also negatively affect mental health and participation in other health behaviours. ${ }^{88}$ Based on an existing scale of neighbourhood disorder, which demonstrated good levels of construct validity and internal consistency/reliability, ${ }^{89}$ four items will assess each of the following characteristics of neighbourhood disorder: physical disorder and physical order, social disorder and social order. These are assessed using the following items: (1) 'My neighbourhood is noisy', (2) 'My neighbourhood is clean', (3), 'There is a lot of crime in my neighbourhood' and (4) 'My neighbourhood is safe' These items will be answered on a five-point scale from strongly disagree (1) to strongly agree (5) and the average responses across the four items will be calculated.

\section{Sample characteristics}

A range of demographic and socioeconomic factors such as age, gender, height and weight, education, income, marital status, occupation, working hours, etc will be assessed. Participants will be asked to also indicate (allowing multiple selection) whether they have been told by a doctor that they have any of the following chronic diseases: arthritis, asthma, cancer, cerebrovascular disease (stroke), chronic obstructive pulmonary disease (emphysema), coronary heart disease (heart attack, angina), type-1-diabetes, type 2 diabetes, high blood pressure, kidney disease, mental illness (depression, anxiety, etc), osteoporosis, irritable bowel syndrome, high cholesterol, or any other disease (to be specified by the participant). In addition, participants will be assessed for morningness or eveningness type ${ }^{90}$ as eveningness types are thought to be more prone to engage in activities that cause social jet-lag, due to misalignments between times of sleep and times of social activity. ${ }^{91}$

\section{Power and sample size}

Meta-analyses of physical activity interventions typically report small to moderate increases in physical activity (Cohen's $d=0.14-0.68) .{ }^{92} 93$ Moreover, poor sleep health, specifically the duration or quality of sleep, has small to moderate magnitude associations with lower physical activity levels. ${ }^{94}$ Meta-analyses of non-pharmacological sleep interventions report small to medium effect sizes for changes in sleep quality (Hedge's $g=0.35$ and Cohen's $d=0.41$ ) in clinical populations, ${ }^{95} 96$ and medium to large effect sizes $(d=0.74)$ in studies using exercise to improve sleep. ${ }^{97}$ Therefore, based on these observations and feasibility data from a previous study, ${ }^{38}$ it was assumed that a three month combined physical activity and sleep intervention that specifically targets both behaviours and leverages the bi-directional relationship between behaviours is likely to produce moderate increases in physical activity $(d=0.45)$ and moderate to large increases in sleep quality $(d=0.65)$. Pre-post correlations were based on preliminary data taken from a trial targeting and measuring changes in physical activity and sleep, ${ }^{38}$ which showed correlations of $r=0.57$ and $r=0.60$ for physical activity and sleep quality, respectively; therefore a pre-post correlation of 0.60 was assumed in the current study. Assuming an alpha of 0.025 (due to measuring two primary outcomes; MVPA and sleep 
quality), power of 0.80 , a moderate effect size $(d=0.45$ for physical activity; $d=0.65$ for sleep) and a pre-post correlation of 0.60 , a total of 60 participants per group will be required for physical activity and 35 per group for sleep quality, the larger sample of which will be used.

Meta-analyses of physical activity and sleep interventions report average drop-out rates of $20 \%,{ }^{93} 95$ however, the majority of web-based trials report drop-out rates that are higher than that. ${ }^{92}$ As there is insufficient information available on attrition in m-health interventions, the sample size for this study will be inflated to account for a 25\% drop-out. Therefore, the total sample size is 80 participants per group or 160 in total. A sample of this size will also be adequately powered to detect mediated effect sizes of small $(\beta=0.14)$ magnitude. ${ }^{98}$ The participant recruitment phase will conclude once 160 complete baseline responses have been obtained.

\section{Analyses}

Analyses will apply the intention-to-treat principle. Analysis of primary outcomes will be blinded to group allocation and overseen by an independent statistician. The primary aim of this study will be to examine differences in physical activity and sleep quality between the intervention group and the control group at the three month primary time point. Between-group differences in physical activity (AAQ minutes) and sleep quality (PSQI) will be estimated using Generalised Linear Mixed Models (GLMM) adjusting for baseline measures of the outcome, including all available data in the analysis. The model will include fixed effects for group, time and their interaction. A random intercept will be used to account for repeated measures on individuals. Separate GLMM will be used to examine changes in physical activity and sleep.

Sensitivity analyses using Pattern Mixture Modelling will be conducted to examine the impact of missing data on outcomes. Where the GLMM assumes data are missing at random, Pattern Mixture Modelling is robust to the assumed pattern of missing data. Group differences in secondary outcome measures will be estimated using the same linear mixed modelling approach, setting an alpha of 0.05 for each outcome. Potential mediators and moderators of intervention efficacy will be examined using established approaches. ${ }^{99}$ Generalised linear mixed models and survival analysis will be used to examine differences in usage patterns.

\section{Ethics and dissemination}

Any type of adverse events reported by study participants that occur in relation to their participation in the study will be recorded and reported to the HREC. This may include events reported by participants, including musculoskeletal injuries associated with the uptake or increase in physical activity or emotional distress due to any survey items of sensitive nature. Great care will be taken to avoid and prevent adverse events and the research team will provide every possible assistance to remediate those events, should they occur. The participant information statement interested individuals will have access to prior to consenting to participate details any potential risks of discomfort associated with participation in the study and provides contact details and information of national support services (eg, Black Dog Institute, Lifeline, etc).

Survey data will be exported directly from Qualtrics as a text file and imported in electronic form for scoring and analysis using statistics software. A detailed database will track participants' progress through the trial including the scheduling of assessments and reminders to complete assessments. Intervention usage will be monitored throughout the trial by BM and MJD by way of the password-protected app database. Given the purpose of the trial, the data to be collected as well as the nature of the intervention, no Data Monitoring Committee will be established. Detailed strategies, including Email/text message reminders will be used to remind participants about upcoming assessments. All Newcastle-based members of the research team (BM, MJD, ATR, RCP) and other associated personnel will have access to the information in both identified and re-identifiable forms. Should statistical analysis advice be sought, access to the data will be granted in re-identifiable form using unique numerical identifiers and access approved by the relevant Ethics Committee.

Print data will be stored in locked filing cabinets accessible only to the research team. Electronic data will be stored on password-protected computers or servers only accessible to the research team. Data will be retained for 15 years in accordance with section 3.1.1 of the Australian Code for the Responsible Conduct of Research and all (paper and electronic) records will be disposed of in accordance with the requirements of the Australian Code for the Responsible Conduct of Research.

Results from the outcome measures will not be presented in a way that adversely affects the confidentiality of participants. The description of participants will not allow identification of individual participants, and individual results and individual names will not be revealed. Final reports and publications will only consist of aggregated results. At the completion of the study, participants will receive a plain English summary of study results. Scientific reports of the main outcomes, secondary outcomes and process evaluation will be submitted to peer-reviewed journals. Results will also be incorporated into student theses and presented at national and international conferences.

\section{DISCUSSION}

It is advised that adults accumulate a weekly minimum of 150 minutes of moderate-intensity physical activity, combined with muscle strengthening activities on two days per week, ${ }^{7}$ and also achieve seven to nine hours of good quality sleep each night. ${ }^{100}$ Engaging in the recommended levels of physical activity and maintaining good sleep health contributes to overall health and well-being through risk reductions associated with chronic diseases such as heart disease and 
type2 diabetes. ${ }^{3-6}$ Engaging in optimal levels of physical activity and sleep health can also positively contribute to long-term weight management, mental health and overall quality of life. ${ }^{101-103}$ Notwithstanding this wide spectrum of benefits, a large proportion of the population does not accumulate sufficient physical activity and/or achieve optimal sleep.

Wide reaching behavioural programmes, such as those offered through m-health interventions, have the potential to elicit the much needed shift of relatively large groups of the population toward levels of physical activity and sleep that meet recommendations. ${ }^{104}$ Multiple behaviour interventions are effective at changing health behaviours ${ }^{19}$ and while m-health interventions as such have been shown to effectively improve physical activity and sleep health as individual behaviours, ${ }^{105} 106$ there is additional evidence from website-based interventions supporting the efficacy of remotely delivered interventions targeting multiple behaviours in combination. ${ }^{107} 108$ To yield positive changes in health behaviour, such interventions need to include educational information, incorporate behaviour change techniques and deliver a level of guidance that endorse the initiation and maintenance of health behaviour change. ${ }^{29}{ }^{109}$ Systematic reviews of the effectiveness of multiple health behaviour interventions suggested that those targeting related behaviours (eg, diet and physical activity) produced greater behaviour change than those targeting unrelated behaviours (eg, smoking and physical activity), ${ }^{110}$ and that specific intervention techniques are necessary for each behaviour. ${ }^{26}$ Physical activity and sleep are suggested to have a bi-directional relationship, ${ }^{16}$ yet no previous RCTs have combined physical activity and sleep in one intervention to utilise the synergistic relationship between physical activity and sleep. The Synergy Study addresses this by targeting both physical activity and sleep, simultaneously, using specific intervention techniques to enhance participants' self-regulatory skills in relation to the two health behaviours and thus, leverages the potential for synergistic improvements in both behaviours. An advantage of this study lies in its mode of delivery, which involves mobile technology and therefore blends into day-to-day life. A key intervention strategy is the use of goal-setting and feedback to promote behaviour change. It seeks to achieve this through the promotion of dynamic goals and action plans, the implementation of a personalised support system further addresses potential barriers (ie, low levels of self-efficacy) that can increase the gap between participant intentions (goals and plans) and behavioural outcomes, ${ }^{111}$ and contribute to long-term behaviour maintenance. This includes knowledge on how to set attainable goals and having strategies in place that facilitate the occurrence of healthy behaviours, despite challenging situations or unfavourable environmental factors. ${ }^{29}$ The structured promotion of goal-setting strategies, combined with action plans that define in detail how an individual will implement the intended behaviour, is known to be effective in changing health behaviours. ${ }^{109}$
Additional strengths of this study include its randomised waitlist controlled study design, which will allow making inferences about the causal links between the intervention and changes in behaviour. The use of remote delivery through a m-health format makes it possible to recruit nationwide and has the potential to be scaled up further including an international version of the programme. Delivering the Synergy Study in multiple countries however, would require further refinement of the contents and adaptation to cross-cultural factors as well as geographical differences. While it is the first aim of this study to test the intervention's efficacy to produce changes in two primary outcomes, the pre-specified secondary outcomes (mental health, quality of life) will give insight into changes in parameters of health and well-being that may be very meaningful to the participant. And finally, this study will generate knowledge on social cognitive determinants of behaviour change relating to sleep health and explore how these factors differ between physical activity and sleep. This will enhance the understanding of underlying mechanisms associated with successful behaviour change in both behaviours and also further the application of social cognitive theories in the multi health behaviour context. The limitations of this study include the study duration, which, although at 6 months is longer than many studies,${ }^{92}$ does not provide insight into longer term changes and behaviour maintenance; and the lack of a comparator condition receiving only the sleep or the physical activity programme to determine the magnitude each intervention component has on its own. It is beyond the scope of this study to test longterm efficacy exceeding six months, but future trials may be encouraged to do this, provided the Synergy Study proves efficacious in the short term with indications of effect retention at the six month time point.

\section{CONCLUSION}

This study protocol provides the rationale and methods associated with the implementation and evaluation of the Synergy Study, a theory-based m-health intervention including personalised support, with the aim to improve physical activity and sleep health in Australian adults. To the authors' knowledge, this study will be the first to simultaneously target changes in these two behaviours, using a sophisticated combination of technologies and evidencebased strategies and test the efficacy of this approach in a randomised controlled trial. Findings from this trial will provide valuable knowledge pertaining to the design of m-health interventions that combine behaviours in a format with wide reach.

\section{Study sponsorship, funding and organisation}

This study was supported in part by funds from a Future Leader Fellowship from the National Heart Foundation of Australia awarded to MJD (ID 100029) as well as a Vanguard Grant from the National Heart Foundation of Australia awarded to MJD (ID 100629). ATR is supported 
by a Wests Scholarship (ID G1201152) and CV (ID 100427) is supported by a Future Leader Fellowship from the National Heart Foundation of Australia.

The trial is sponsored by the University of Newcastle and will be coordinated independently of the study sponsor and funder by the Priority Research Centre for Physical Activity and Nutrition, University of Newcastle, Australia and managed by project lead BM and overseen by chief investigator MJD. The study funder and sponsor will have no role in the conducting or evaluation of the trial, nor did the study funder and sponsor have any authority over the study design, collection, management, analysis and interpretation of data, the preparation of manuscripts or the submission of reports.

\section{Author affiliations}

${ }^{1}$ Priority Research Centre for Physical Activity and Nutrition, University of Newcastle, Newcastle, Australia

${ }^{2}$ School of Medicine and Public Health, Faculty of Health and Medicine, University of Newcastle, Callaghan, Australia

${ }^{3}$ School of Education, Faculty of Education and Arts, University of Newcastle, Callaghan, Australia

${ }^{4}$ School of Health, Medical and Applied Science, Physical Activity Research Group, Central Queensland University, Rockhampton, Australia

${ }^{5}$ Centre for Research on Exercise, Physical Activity and Health, School of Human Movement and Nutrition Studies, The University of Queensland, Brisbane, Australia

Contributors All authors meet ICMJE criteria for authorship in that they have contributed substantially to the conceptual design; or the processes of data collection, analysis or interpretation; the drafts and revisions of the study protocol and manuscript; granted approval of the final version of the study protocol and acknowledged their accountability with regard to the integrity and accuracy of this study protocol. In detail, the first author of this protocol (BM) will be responsible for administrative and managerial procedures related to all phases of the trial, which will be supervised by MJD and RCP. ATR will fulfil this role, in the case of BM's temporary illness or absence. BM, MJD, ATR and RCP contributed to the development of study materials, MJD, CV and WJB have contributed to the conceptual design of the trial and all authors (BM, RCP, ATR, CV, WJB and MJD) contributed to the writing of the protocol and will be involved in the interpretation of results, the evaluation of the trial and dissemination of study findings.

Funding ATR is supported by a Wests Scholarship (ID G1201152). MJD (ID 100029) and CV (ID 100427) are supported by a Future Leader Fellowship from the National Heart Foundation of Australia.

\section{Competing interests None declared.}

Patient consent Detail has been removed from this case description/these case descriptions to ensure anonymity. The editors and reviewers have seen the detailed information available and are satisfied that the information backs up the case the authors are making.

Ethics approval Full ethical approval was obtained from the Human Research Ethics Committee of The University of Newcastle, Australia (Approval Number: H-2016-0181).

Provenance and peer review Not commissioned; externally peer reviewed.

Author note Any amendments to the study protocol will be submitted to the Human Research Ethics Committee (HREC)and updated on the trial register (ANZCTR) once full ethical approval has been obtained.

Open Access This is an Open Access article distributed in accordance with the Creative Commons Attribution Non Commercial (CC BY-NC 4.0) license, which permits others to distribute, remix, adapt, build upon this work non-commercially, and license their derivative works on different terms, provided the original work is properly cited and the use is non-commercial. See: http://creativecommons.org/ licenses/by-nc/4.0/

(C) Article author(s) (or their employer(s) unless otherwise stated in the text of the article) 2018. All rights reserved. No commercial use is permitted unless otherwise expressly granted.
REFERENCES

1 Schmid D, Ricci C, Leitzmann MF. Associations of objectively assessed physical activity and sedentary time with allcause mortality in US adults: the NHANES study. PLoS One 2015;10:e0119591.

2 Gallicchio L, Kalesan B. Sleep duration and mortality: a systematic review and meta-analysis. J Sleep Res 2009;18:148-58.

3 Shiroma EJ, Lee IM. Physical activity and cardiovascular health: lessons learned from epidemiological studies across age, gender, and race/ethnicity. Circulation 2010;122:743-52.

4 Hoevenaar-Blom MP, Spijkerman AM, Kromhout D, et al. Sleep duration and sleep quality in relation to 12-year cardiovascular disease incidence: the MORGEN study. Sleep 2011;34:1487-92.

5 Shan Z, Ma H, Xie M, et al. Sleep duration and risk of type 2 diabetes: a meta-analysis of prospective studies. Diabetes Care 2015;38:529-37.

6 Aune D, Norat T, Leitzmann M, et al. Physical activity and the risk of type 2 diabetes: a systematic review and dose-response metaanalysis. Eur J Epidemiol 2015;30:529-42.

7 The Department of Health. Australia's physical activity and sedentary behaviour guidelines for adults (18-64 years. Canberra: Commonwealth of Australia, 2014. http://www.webcitation.org/ 6pseaziKS

8 Buysse DJ. Sleep health: can we define it? Does it matter? Sleep 2014;37:9-17.

9 World Health Organisation. Physical activity: Fact sheet. Geneva: World Health Organization, 2017.

10 Hoyos C, Glozier N, Marshall NS. Recent evidence on worldwide trends on sleep duration. Curr Sleep Med Rep 2015;1:195-204.

11 Buman MP, Phillips BA, Youngstedt SD, et al. Does nighttime exercise really disturb sleep? Results from the 2013 national sleep foundation sleep in America poll. Sleep Med 2014;15:755-61.

12 Duncan MJ, Kline CE, Rebar AL, et al. Greater bed- and waketime variability is associated with less healthy lifestyle behaviors: a cross-sectional study. Z Gesundh Wiss 2016;24:31-40.

13 Bixler E. Sleep and society: an epidemiological perspective. Sleep Med 2009;10:S3-6.

14 Rayward AT, Duncan MJ, Brown WJ, et al. A cross-sectional cluster analysis of the combined association of physical activity and sleep with sociodemographic and health characteristics in mid-aged and older adults. Maturitas 2017;102:56-61.

15 Prochaska JJ, Prochaska JO. A review of multiple health behavior change interventions for primary prevention. Am J Lifestyle Med 2011;5:208-21.

16 Kline CE. The bidirectional relationship between exercise and sleep: Implications for exercise adherence and sleep improvement. Am J Lifestyle Med 2014;8:375-9.

17 Lopresti AL, Hood SD, Drummond PD. A review of lifestyle factors that contribute to important pathways associated with major depression: diet, sleep and exercise. J Affect Disord 2013;148:12-27.

18 Chennaoui M, Arnal PJ, Sauvet F, et al. Sleep and exercise: a reciprocal issue? Sleep Med Rev 2015;20:59-72.

19 James E, Freund M, Booth A, et al. Comparative efficacy of simultaneous versus sequential multiple health behavior change interventions among adults: A systematic review of randomised trials. Prev Med 2016;89:211-23.

20 King K, Meader N, Wright K, et al. Characteristics of interventions targeting multiple lifestyle risk behaviours in adult populations: a systematic scoping review. PLoS One 2015;10:e0117015.

21 Meader N, King K, Wright K, et al. Multiple risk behavior interventions: meta-analyses of RCTs. Am J Prev Med 2017;53:e19-30.

22 Irish LA, Kline CE, Gunn HE, et al. The role of sleep hygiene in promoting public health: A review of empirical evidence. Sleep Med Rev 2015;22:23-36.

23 Brown FC, Buboltz WC, Soper B. Development and evaluation of the Sleep Treatment and Education Program for Students (STEPS). J Am Coll Health 2006;54:231-7.

24 Kakinuma M, Takahashi M, Kato N, et al. Effect of brief sleep hygiene education for workers of an information technology company. Ind Health 2010;48:758-65.

25 Marshall AL, Leslie ER, Bauman AE, et al. Print versus website physical activity programs: a randomized trial. Am J Prev Med 2003;25:88-94.

26 Mc Sharry J, Olander EK, French DP. Do single and multiple behavior change interventions contain different behavior change techniques? A comparison of interventions targeting physical activity in obese populations. Health Psychol 2015;34:960-5. 
27 Prestwich A, Sniehotta FF, Whittington C, et al. Does theory influence the effectiveness of health behavior interventions? Metaanalysis. Health Psychol 2014;33:465-74.

28 Plotnikoff RC, Costigan SA, Karunamuni N, et al. Social cognitive theories used to explain physical activity behavior in adolescents: a systematic review and meta-analysis. Prev Med 2013;56:245-53.

29 Bandura A. Health promotion from the perspective of social cognitive theory. Psychol Health 1998;13:623-49.

30 Grandner MA, Jackson NJ, Izci-Balserak B, et al. Social and behavioral determinants of perceived insufficient sleep. Front Neurol 2015;6:112.

31 Young MD, Plotnikoff RC, Collins CE, et al. Social cognitive theory and physical activity: a systematic review and meta-analysis. Obes Rev 2014;15:983-95.

32 Rovniak LS, Anderson ES, Winett RA, et al. Social cognitive determinants of physical activity in young adults: a prospective structural equation analysis. Ann Behav Med 2002;24:149-56.

33 Pew Research Center. Smartphone Ownership and Internet Usage Continues to Climb in Emerging Economies. In: Poushter J, ed. USA: Pew Research, 2016.

34 Schulz KF, Altman DG, Moher D, et al. CONSORT 2010 statement: updated guidelines for reporting parallel group randomized trials. Ann Intern Med 2010;152:726-32.

35 Eysenbach G. CONSORT-EHEALTH Group. CONSORT-EHEALTH: improving and standardizing evaluation reports of Web-based and mobile health interventions. J Med Internet Res 2011;13:e126.

36 Duncan M, Murawski B, Short CE, et al. Activity trackers implement different behavior change techniques for activity, sleep, and sedentary behaviors. Interact J Med Res 2017;6:e13

37 Lyons EJ, Lewis ZH, Mayrsohn BG, et al. Behavior change techniques implemented in electronic lifestyle activity monitors: a systematic content analysis. J Med Internet Res 2014;16:e192.

38 Duncan MJ, Vandelanotte C, Trost SG, et al. Balanced: randomised trial examining the efficacy of two self-monitoring methods for an app-based multi-behaviour intervention to improve physical activity, sitting and sleep in adults. BMC Public Health 2016;16:670.

39 De Souto Barreto P. Global health agenda on non-communicable diseases: has WHO set a smart goal for physical activity? BMJ 2015;350:h23

40 Dennison L, Morrison L, Conway G, et al. Opportunities and challenges for smartphone applications in supporting health behavior change: qualitative study. J Med Internet Res 2013;15:e86.

41 Locke EA, Latham GP. A theory of goal setting task and performance. Englewood Cliffs, NJ, US: Prentice-Hall, Inc, 1990.

42 Health Direct. Relaxation techniques for stress relief. http://www. webcitation.org/6pMQB1x2c (accessed 30 Mar 2017).

43 Black Dog Institute. Mindfulness in everyday life - with GP notes. http://www.webcitation.org/6pMTDbhpU (accessed 30 Mar 2017).

44 Black Dog Institute. Mindfulness in everyday life. http://www. webcitation.org/6pMTOm3Dj (accessed 30 Mar 2017).

45 Sleep Health Foundation. How much sleep do you really need? 2011 http://www.webcitation.org/6pse5ltGe (accessed 15 Feb 2017)

46 Doig GS, Simpson F. Randomization and allocation concealment: a practical guide for researchers. J Crit Care 2005;20:187-91. discussion 91-3.

47 Australian Institute of Health and Welfare. The active Australia survey: a guide and manual for implementation, analysis and reporting. Canberra: AlHW, 2003.

48 Brown WJ, Burton NW, Marshall AL, et al. Reliability and validity of a modified self-administered version of the Active Australia physical activity survey in a sample of mid-age women. Aust N Z J Public Health 2008;32:535-41.

49 Reeves MM, Marshall AL, Owen N, et al. Measuring physical activity change in broad-reach intervention trials. J Phys Act Health 2010;7:194-202.

50 Buysse DJ, Reynolds CF, Monk TH, et al. The Pittsburgh Sleep Quality Index: a new instrument for psychiatric practice and research. Psychiatry Res 1989;28:193-213.

51 Ait-Aoudia M, Levy PP, Bui E, et al. Validation of the French version of the pittsburgh sleep quality index addendum for posttraumatic stress disorder. Eur J Psychotraumatol 2013;4:19298.

52 Lewith GT, Godfrey AD, Prescott P. A single-blinded, randomized pilot study evaluating the aroma of Lavandula augustifolia as a treatment for mild insomnia. J Altern Complement Med 2005;11:631-7.

53 Nebes RD, Buysse DJ, Halligan EM, et al. Self-reported sleep quality predicts poor cognitive performance in healthy older adults. J Gerontol B Psychol Sci Soc Sci 2009;64:180-7.
54 Backhaus J, Junghanns K, Broocks A, et al. Test-retest reliability and validity of the Pittsburgh Sleep Quality Index in primary insomnia. J Psychosom Res 2002;53:737-40.

55 Littner M, Hirshkowitz M, Kramer M, et al. Practice parameters for using polysomnography to evaluate insomnia: an update. Sleep 2003;26:754-60.

56 Rogers LQ, Courneya KS, Oster RA, et al. Physical activity and sleep quality in breast cancer survivors: a randomized trial. Med Sci Sports Exerc 2017;49:2009-15.

57 Duncan MJ, Kline CE, Vandelanotte C, et al. Cross-sectional associations between multiple lifestyle behaviors and health-related quality of life in the 10,000 Steps cohort. PLoS One 2014;9:e94184.

58 Busija L, Pausenberger E, Haines TP, et al. Adult measures of general health and health-related quality of life: Medical Outcomes Study Short Form 36-Item (SF-36) and Short Form 12-Item (SF-12) Health Surveys, Nottingham Health Profile (NHP), Sickness Impact Profile (SIP), Medical Outcomes Study Short Form 6D (SF-6D), Health Utilities Index Mark 3 (HUI3), Quality of Well-Being Scale (QWB), and Assessment of Quality of Life (AQoL). Arthritis Care Res 2011;63:S383-412.

59 Henry JD, Crawford JR. The short-form version of the Depression Anxiety Stress Scales (DASS-21): construct validity and normative data in a large non-clinical sample. $\mathrm{Br} J$ Clin Psychol 2005;44:227-39.

60 Bampton EA, Johnson ST, Vallance JK. Profiles of resistance training behavior and sedentary time among older adults: Associations with health-related quality of life and psychosocial health. Prev Med Rep 2015;2:773-6.

61 Chau JY, van der Ploeg HP, Dunn S, et al. A tool for measuring workers' sitting time by domain: the workforce sitting questionnaire. Br J Sports Med 2011;45:1216-22.

62 Prince SA, Saunders TJ, Gresty K, et al. A comparison of the effectiveness of physical activity and sedentary behaviour interventions in reducing sedentary time in adults: a systematic review and meta-analysis of controlled trials. Obes Rev 2014;15:905-19.

63 Monk TH, Buysse DJ, Kennedy KS, et al. Measuring sleep habits without using a diary: the sleep timing questionnaire. Sleep 2003;26:208-12.

64 Morin CM, Belleville G, Bélanger L, et al. The Insomnia Severity Index: psychometric indicators to detect insomnia cases and evaluate treatment response. Sleep 2011;34:601-8.

65 Johns MW. A new method for measuring daytime sleepiness: the epworth sleepiness scale. Sleep 1991;14:540-5.

66 Eastin MS, LaRose R. Internet self-efficacy and the psychology of the digital divide. J Comput Mediat Commun 2006;6.

67 Coursaris CK, van Osch W. A Cognitive-Affective Model of Perceived User Satisfaction (CAMPUS): the complementary effects and interdependence of usability and aesthetics in IS design. Inf Manage 2016;53:252-64.

68 Kolt GS, Rosenkranz RR, Vandelanotte C, et al. Using Web 2.0 applications to promote health-related physical activity: findings from the WALK 2.0 randomised controlled trial. Br J Sports Med 2017;51:1433-40.

69 Guertler D, Vandelanotte C, Kirwan M, et al. Engagement and nonusage attrition with a free physical activity promotion program: the case of 10,000 Steps Australia. J Med Internet Res 2015; $17:$ e176.

70 Brooke J. SUS - a quick and dirty usability scale. In: Jordan PW, Thomas B, Weerdmeester BA, eds. Usability evaluation in industry. London: Taylor and Francis, 1996:194.

71 Vandelanotte C, Short C, Plotnikoff RC, et al. TaylorActiveExamining the effectiveness of web-based personally-tailored videos to increase physical activity: a randomised controlled trial protocol. BMC Public Health 2015;15:1020.

72 Plotnikoff RC, Lubans DR, Penfold CM, et al. Testing mediator variables in a physical activity intervention for women with type 2 diabetes. Psychol Sport Exerc 2014;15:1-8.

73 Plotnikoff RC, Lippke S, Courneya KS, et al. Physical activity and social cognitive theory: a test in a population sample of adults with type 1 or type 2 diabetes. Appl Psychol 2008;57:628-43.

74 Rogers LQ, Humphries MC, Davis H, et al. Predictors of physical activity among internal medicine clinic patients. Med Sci Sports Exerc 1998;30:96.

75 Dewar DL, Lubans DR, Plotnikoff RC, et al. Development and evaluation of social cognitive measures related to adolescent dietary behaviors. Int J Behav Nutr Phys Act 2012;9:36.

76 Liebreich T, Plotnikoff RC, Courneya KS, et al. Diabetes NetPLAY: a physical activity website and linked email counselling randomized intervention for individuals with type 2 diabetes. Int J Behav Nutr Phys Act 2009;6:18. 
77 Alexander A, Bergman P, Hagströmer M, et al. IPAQ environmental module; reliability testing. J Public Health 2006;14:76-80.

78 Rhodes RE, Hunt Matheson D, Mark R. Evaluation of social cognitive scaling response options in the physical activity domain. Meas Phys Educ Exerc Sci 2010;14:137-50.

79 Trinh L, Plotnikoff RC, Rhodes RE, et al. Correlates of physical activity in a population-based sample of kidney cancer survivors: an application of the theory of planned behavior. Int J Behav Nutr Phys Act 2012;9:96.

80 Schwarzer R, Luszczynska A. Self-Beliefs and Self-Regulation in Health Behavior Change. In: Guay F, Marsh H, Mclnerney DM, eds. Self-concept, motivation and identity: underpinning success with research and practice. Charlotte NC: Information Age Publishing Inc, 2015:201-24.

81 Kor K, Mullan BA. Sleep hygiene behaviours: an application of the theory of planned behaviour and the investigation of perceived autonomy support, past behaviour and response inhibition. Psychol Health 2011;26:1208-24.

82 Rebar AL, Dimmock JA, Jackson B, et al. A systematic review of the effects of non-conscious regulatory processes in physical activity. Health Psychol Rev 2016;10:395-407.

83 Gardner B, Abraham C, Lally P, et al. Towards parsimony in habit measurement: testing the convergent and predictive validity of an automaticity subscale of the Self-Report Habit Index. Int J Behav Nutr Phys Act 2012;9:102.

84 Mastin DF, Bryson J, Corwyn R. Assessment of sleep hygiene using the Sleep Hygiene Index. J Behav Med 2006;29:223-7.

85 Duncan M, Mummery K. Psychosocial and environmental factors associated with physical activity among city dwellers in regional Queensland. Prev Med 2005;40:363-72.

86 Hale L, Hill TD, Friedman E, et al. Perceived neighborhood quality, sleep quality, and health status: evidence from the Survey of the Health of Wisconsin. Soc Sci Med 2013;79:16-22.

87 Johnson DA, Simonelli G, Moore K, et al. The neighborhood social environment and objective measures of sleep in the multi-ethnic study of atherosclerosis. Sleep 2017;40.

88 Hill TD, Ross CE, Angel RJ. Neighborhood disorder, psychophysiological distress, and health. J Health Soc Behav 2005;46:170-86.

89 Hale L, Hill TD, Burdette AM. Does sleep quality mediate the association between neighborhood disorder and self-rated physical health? Prev Med 2010;51:275-8.

90 Horne JA, Ostberg O. A self-assessment questionnaire to determine morningness-eveningness in human circadian rhythms. Int $J$ Chronobiol 1976;4:97-110.

91 Wittmann M, Dinich J, Merrow M, et al. Social jetlag: misalignment of biological and social time. Chronobiol Int 2006;23:497-509.

92 Davies CA, Spence JC, Vandelanotte C, et al. Meta-analysis of internet-delivered interventions to increase physical activity levels. Int J Behav Nutr Phys Act 2012;9:52.

93 Kang M, Marshall SJ, Barreira TV, et al. Effect of pedometer-based physical activity interventions: a meta-analysis. Res Q Exerc Sport 2009;80:648-55.

94 Bromley LE, Booth JN, Kilkus JM, et al. Sleep restriction decreases the physical activity of adults at risk for type 2 diabetes. Sleep 2012;35:977-84.
95 Ho FY, Chung KF, Yeung WF, et al. Self-help cognitive-behavioral therapy for insomnia: a meta-analysis of randomized controlled trials. Sleep Med Rev 2015;19:17-28.

96 Cheng SK, Dizon J. Computerised cognitive behavioural therapy for insomnia: a systematic review and meta-analysis. Psychother Psychosom 2012;81:206-16.

97 Kredlow MA, Capozzoli MC, Hearon BA, et al. The effects of physical activity on sleep: a meta-analytic review. J Behav Med 2015;38:427-49.

98 Fritz MS, Mackinnon DP. Required sample size to detect the mediated effect. Psychol Sci 2007;18:233-9.

99 Imai K, Keele L, Tingley D. A general approach to causal mediation analysis. Psychol Methods 2010;15:309-34.

100 Hirshkowitz M, Whiton K, Albert SM, et al. National Sleep Foundation's sleep time duration recommendations: methodology and results summary. Sleep Health 2015;1:40-3.

101 Swift DL, Johannsen NM, Lavie CJ, et al. The role of exercise and physical activity in weight loss and maintenance. Prog Cardiovasc Dis 2014;56:441-7.

102 Stanton R, Happell B, Reaburn P. The mental health benefits of regular physical activity, and its role in preventing future depressive illness. Nursing 2014;45:45.

103 Beccuti G, Pannain S. Sleep and obesity. Curr Opin Clin Nutr Metab Care 2011;14:402-12.

104 Owen N, Sparling PB, Healy GN, et al. Sedentary behavior: emerging evidence for a new health risk. Mayo Clin Proc 2010;85:1138-41.

105 Müller AM, Alley S, Schoeppe S, et al. The effectiveness of e-\& mHealth interventions to promote physical activity and healthy diets in developing countries: A systematic review. Int J Behav Nutr Phys Act 2016;13:109.

106 Shin JC, Kim J, Grigsby-Toussaint D. Mobile Phone Interventions for Sleep Disorders and Sleep Quality: Systematic Review. JMIR Mhealth Uhealth 2017:5:e131.

107 Schulz DN, Kremers SP, Vandelanotte C, et al. Effects of a webbased tailored multiple-lifestyle intervention for adults: a two-year randomized controlled trial comparing sequential and simultaneous delivery modes. J Med Internet Res 2014;16:e26.

108 Parekh S, Vandelanotte C, King D, et al. Improving diet, physical activity and other lifestyle behaviours using computer-tailored advice in general practice: a randomised controlled trial. Int J Behav Nutr Phys Act 2012;9:108.

109 Samdal GB, Eide GE, Barth T, et al. Effective behaviour change techniques for physical activity and healthy eating in overweight and obese adults; systematic review and meta-regression analyses. Int J Behav Nutr Phys Act 2017;14:42

110 Yin HQ, Prochaska JO, Rossi JS, et al. Treatment-enhanced paired action contributes substantially to change across multiple health behaviors: secondary analyses of five randomized trials. Trans/ Behav Med 2013;3:62-71.

111 Sniehotta FF, Scholz U, Schwarzer R. Bridging the intentionbehaviour gap: Planning, self-efficacy, and action control in the adoption and maintenance of physical exercise. Psychol Health 2005;20:143-60.

112 Michie S, Ashford S, Sniehotta FF, et al. A refined taxonomy of behaviour change techniques to help people change their physical activity and healthy eating behaviours: the CALO-RE taxonomy. Psychol Health 2011;26:1479-98. 